\title{
High quality factor Al2O3 microring resonators for on-chip sensing applications
}

de Goede, M., Dijkstra, M., Obregón Núñez, R., Martínez, E., García-Blanco, S.

M. de Goede, M. Dijkstra, R. Obregón Núñez, E. Martínez, S. M. GarcíaBlanco, "High quality factor Al2O3 microring resonators for on-chip sensing applications," Proc. SPIE 10535, Integrated Optics: Devices, Materials, and Technologies XXII, 1053504 (23 February 2018); doi: 10.1117/12.2290004

SPIE. Event: SPIE OPTO, 2018, San Francisco, California, United States 


\title{
High quality factor $\mathrm{Al}_{2} \mathrm{O}_{3}$ microring resonators for on-chip sensing applications
}

\author{
M. de Goede*a ${ }^{*}$, M. Dijkstra ${ }^{\mathrm{a}}$, R. Obregón Núñez ${ }^{\mathrm{b}}$, E. Martínez $^{\mathrm{b}}, \mathrm{S}$. M. García-Blanco ${ }^{\mathrm{a}}$ \\ ${ }^{a}$ Optical Sciences Group, MESA+ Institute for Nanotechnology, University of Twente, P.O. Box 217, 7500 \\ AE Enschede, The Netherlands; ${ }^{b}$ Biomimetic Systems for Cell Engineering, Institute for Bioengineering of \\ Catalonia, University of Barcelona, C/ Baldiri Reixac 15-21, 08028 Barcelona, Spain
}

\begin{abstract}
Microring resonators find many applications for on-chip integrated optical sensors. Their spectral response contains resonance dips that shift due to variations of the optical path length of the microring probed. Numerous examples of such microring resonator sensors in the $\mathrm{SOI}, \mathrm{Si}_{3} \mathrm{~N}_{4}$ and $\mathrm{SiON}$ waveguide technologies have been reported for the detection of bulk refractive index variations and the label-free detection of biomarkers. $\mathrm{Al}_{2} \mathrm{O}_{3}$ is an alternative waveguide technology that exhibits low optical propagation losses, is transparent over a large spectral range extending from the visible to the mid-IR and permits co-doping with active rare-earth ions, which enables the co-integration of active devices on the chip. In this work an $\mathrm{Al}_{2} \mathrm{O}_{3}$ microring resonator sensor was developed for the label-free detection of protein biomarkers. The uncladded microring with a radius of $200 \mu \mathrm{m}$ had a measured quality factor of $3.2 \times 10^{5}$ at $1550 \mathrm{~nm}$. Submerging the devices in water decreased the quality factor to $45 \times 10^{3}$. This corresponds with propagation losses in the rings of $0.6 \mathrm{~dB} / \mathrm{cm}$ and $5.7 \mathrm{~dB} / \mathrm{cm}$ respectively. The bulk refractive index sensitivity of the sensor was determined by flowing $\mathrm{NaCl}$ dissolved in water in different concentrations. A sensitivity of $102.3 \pm 0.5 \mathrm{~nm} / \mathrm{RIU}$ with a corresponding limit of detection of $1.6 \times 10^{-6}$ RIU was demonstrated for TM polarized light. High affinity human monoclonal antibodies mAb S100A4 were immobilized on the sensor to detect the S100A4 protein biomarker down to $12 \mathrm{nM}$ concentrations. These results demonstrate the feasibility of this material for label-free optical biosensors.
\end{abstract}

Keywords: $\mathrm{Al}_{2} \mathrm{O}_{3}$, microring resonator, sensing, biomarker.

\section{INTRODUCTION}

Waveguide microring resonators are numerously used for on-chip integrated optical sensors. Their wavelength dependent transmission spectrum contains multiple dips whenever light inside the microring resonator has a roundtrip phase multiple of $2 \pi$ (i.e., the resonance condition). Upon a change in the refractive index of the microring resonator environment, the accumulated roundtrip phase varies, resulting in a shift of the resonance wavelength. The wavelength shift of a resonance can be used as a sensor to detect refractive index variations of its exterior. This type of sensors are excellent candidates for label-free biosensors due to their strong optical interaction with the analyte, high quality factor, compact footprint, and potential for large-scale fabrication ${ }^{1}$.

Many microring resonator based biosensors were realized with silicon-based waveguide technologies. For instance, high sensitivities for both bulk refractive index and surface sensing were already demonstrated for silicon-on-insulator ${ }^{2}$ (SOI), $\mathrm{Si}_{3} \mathrm{~N}_{4}{ }^{3}$, and $\mathrm{SiON}^{4}$ microring resonators. $\mathrm{Al}_{2} \mathrm{O}_{3}$ is an alternative technology that can be used for this type of sensors. Waveguides fabricated in this material have low propagation losses over a large spectral range extending from the visible to the mid-IR ${ }^{5,6}$ and rare-earth ion dopants can be introduced to provide gain and achieve lasers ${ }^{7,8}$. Furthermore, on this platform active sensors were already realized that could detect small particles from a change of the frequency of the beat note of a dual-lasing system at a wavelength of $1 \mu \mathrm{m}^{9}$. This demonstrates that the $\mathrm{Al}_{2} \mathrm{O}_{3}$ technology is suited for active, laser-based biosensors with simple detection schemes.

In this work, the first demonstration of $\mathrm{Al}_{2} \mathrm{O}_{3}$-based microring resonator sensors is presented. The sensor was designed and fabricated for on-chip bulk refractive index and surface sensing. High quality factors were achieved for TE and TM polarizations for both air a water cladded devices. A microring resonator submerged in water was used for bulk refractive index sensing of different salt concentrations dissolved in DI water. The surface of the microring resonator was functionalized to immobilize antibodies. This was followed by the detection of clinically relevant biomarkers down to a 
limit of detection of $12 \mathrm{nM}$. These results, together with the possibility of using rare-earth ion doped $\mathrm{Al}_{2} \mathrm{O}_{3}$ for active applications and gain at wavelengths around $1 \mu \mathrm{m}$, motivate the use of this material for on-chip integrated sensors.

\section{MICRORING RESONATOR DESIGN AND FABRICATION}

The microring resonator sensor was designed for optimal sensing performance. To this end the parameters requiring design were the ring and bus waveguide cross sections, the microring bend radius, and the coupling gap between ring and bus waveguide. These parameters were chosen to ensure resonances with a high quality factor $Q$ that are critically coupled. The former requirement ensures a small full width at half maximum for precise resonance wavelength determination. The latter enhances the fitting of the resonance dip. Furthermore, the sensor should be as sensitive as possible in transducing the applied bulk refractive index variation into a shift of resonance wavelength. The sensitivity $S$ is a measure of how much the resonance wavelength shifts for a variation of the refractive index of the top cladding and it is given by ${ }^{10}$ :

$$
S=\frac{\lambda}{n_{g}} \frac{\partial n_{e f f}}{\partial n}
$$

where $\lambda$ is the resonance wavelength, $n_{g}$ the group index, $n_{\text {eff }}$ the effective index of the mode, and $n$ the refractive index of the top cladding with dimensionless units of refractive index unit (RIU). The derivative of $n_{\text {eff }}$ with respect to $n$ scales with the modal power inside the top cladding sensing region. Thus, achieving a high $S$ amounts to choosing the cross section of the waveguide such that a large fraction of the optical power travels in the top cladding.

Another sensing performance parameter of importance is the limit of detection (LOD). The LOD characterizes the smallest variation of bulk refractive that can still be reliably measured by the sensor and is given by:

$$
\mathrm{LOD}=\frac{3 \varepsilon}{S}
$$

where $\varepsilon$ is the uncertainty or signal noise in the determination of the resonance wavelength ${ }^{11}$.

Optimal sensing performance requires a high $S$ and a low LOD for the best sensor performance. This can be achieved by reducing the waveguide cross section to increase the modal overlap of the waveguide mode with the sensing region to increase $S$. However, this typically increases the mode propagation losses due to increased bend radiation loss and absorption and scattering losses in the sensing medium, resulting in a reduced $Q$. Since a low $Q$ increases the full width at half maximum of the resonance dip, the precision of the determination of the resonance wavelength decreases and increases $\varepsilon$. Therefore, the waveguide cross section and microring radius need to be optimized for both high $S$ and $Q$.

The geometric parameters of the microring resonator were determined based on this criteria. A finite element mode solver was utilized to determine the bend radiation losses, the power coupling from the bus waveguide to the microring resonator, and the modal overlap of the field with the sensing region. The design was performed for operation in an aqueous environment at a central wavelength of $1550 \mathrm{~nm}$. At this wavelength the bottom cladding of $\mathrm{SiO}_{2}$, waveguide material $\mathrm{Al}_{2} \mathrm{O}_{3}$ and the top cladding of water have refractive indices of respectively 1.46, 1.65 and $1.33 \mathrm{RIU}$. The optimum parameters of the ring resonator were determined to be a waveguide cross section of $2.0 \times 0.75 \mu \mathrm{m}^{2}$ for both the bus and microring waveguide, a coupling gap of $0.8 \mu \mathrm{m}$ between these, and a microring radius of $200 \mu \mathrm{m}$. Figure 1a displays the designed waveguide cross section with the calculated $\mathrm{x}$-component of the fundamental TE mode field.

The microring resonator sensor was fabricated with the designed geometrical parameters. First, an $\mathrm{Al}_{2} \mathrm{O}_{3}$ layer was reactively sputtered on a $\mathrm{SiO}_{2}$ substrate with an AJA ATC 1500 sputter coater. On this layer waveguides and microring resonators were patterned with contact UV lithography and subsequently etched with an Oxford Plasmalab System 100 inductively coupled plasma reactive ion etcher. Figure $1 \mathrm{~b}$ shows a resulting microring resonator excited with green light and Figure 1c shows the coupling gap between the bus waveguide and ring resonator. Using a shadow mask, a $\mathrm{SiO}_{2}$ top cladding was locally deposited to cover the bus waveguide but leave the microring resonator open to the aqueous environment for sensing. Small chips were diced containing the microring resonator sensor. Finally, a PDMS microfluidic device was bonded to the chip to allow addressing the sensor with various liquids, as shown in Figure 1d. The microfluidic device contains a channel with width of $600 \mu \mathrm{m}$ and height of $50 \mu \mathrm{m}$. 
(a)

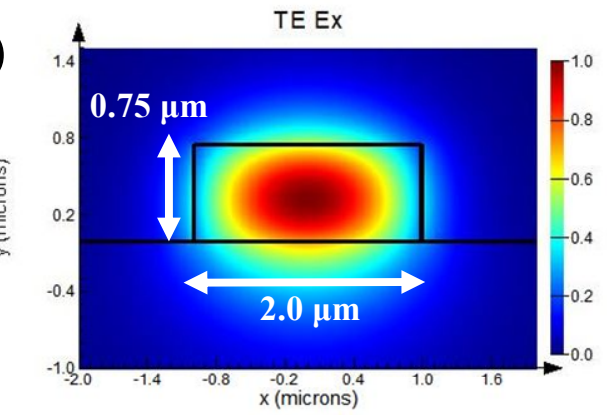

(c)

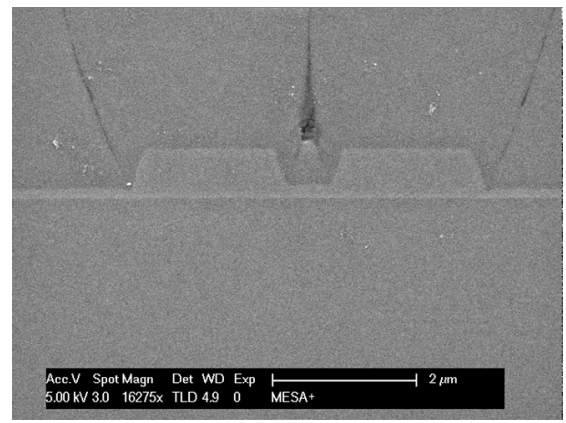

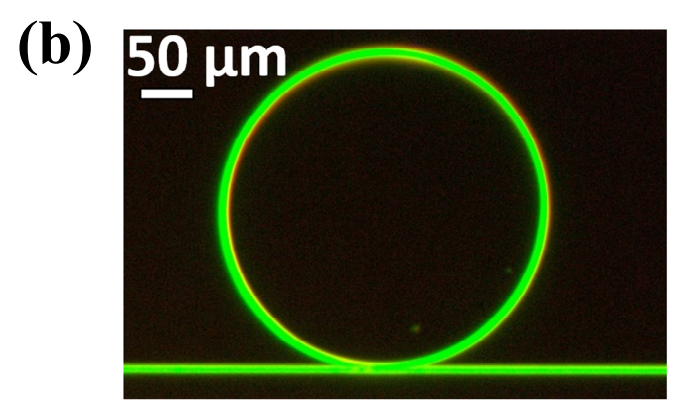

(d)

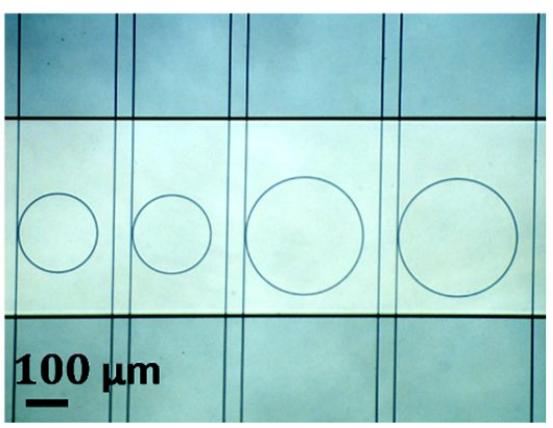

Figure 1. $\mathrm{Al}_{2} \mathrm{O}_{3}$ microring resonator design and fabrication. (a), Designed waveguide cross section together with $\mathrm{x}$ component of fundamental TE mode. (b), Optical microscope image of $\mathrm{Al}_{2} \mathrm{O}_{3}$ microring resonator under illumination. (c), Electron microscope image of the waveguide coupling region. (d), Microfluidic channel bonded over $\mathrm{Al}_{2} \mathrm{O}_{3}$ microring resonator sensors.

\section{MICRORING CHARACTERIZATION}

The $\mathrm{Al}_{2} \mathrm{O}_{3}$ microring resonators were characterized by wavelength dependent transmission measurements. From these, the resonator losses, finesse $F$ and $Q$ are determined. To this end light from an Agilent 81646 tunable laser was guided through a polarization maintaining fiber and butt coupled at the input of the chip containing the sensor. The transmitted light was collected at the output of the chip with another polarization maintaining fiber and guided to a power meter. To enhance the coupling to the chip index matching fluid was used. Both TE and TM polarized light was used. The scanning resolution was $1 \mathrm{pm}$.

The normalized transmission spectrum of the sensor for TE polarized light and a cladding of air is shown in Figure 2a. The resonance dips in this spectra were then fitted to a Lorentzian function, as shown in Figure 2b. From this fit $F, Q$, and the propagation loss were determined, for both TE and TM polarized light for the microring resonator with a cladding of air and water. This was done at $\lambda=1565 \mathrm{~nm}$, such that the water absorption losses are minimal while still remaining in the optical C-band. The determined resonator loss, $F$ and $Q$ are summarized in Table 1. For a cladding of air TM polarized light has three times higher propagation losses than TE polarized light. This could be explained by increased bend radiation losses due to the smaller confinement of the TM mode. Furthermore, submerging the microring resonators in water significantly increases the propagation losses mainly due to water absorption in the near-infrared region. 
(a)

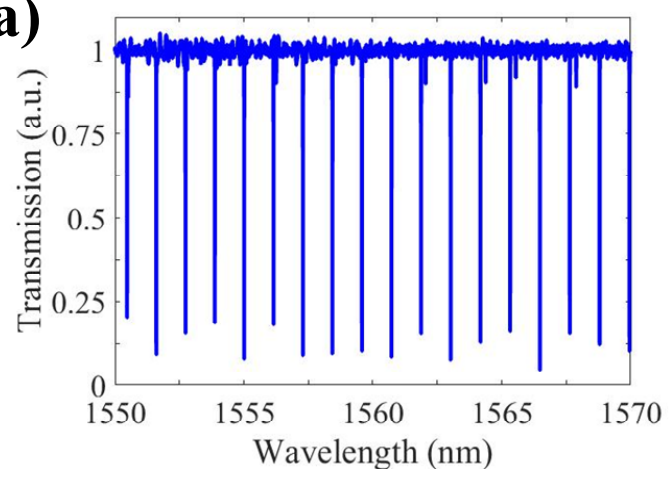

(b)

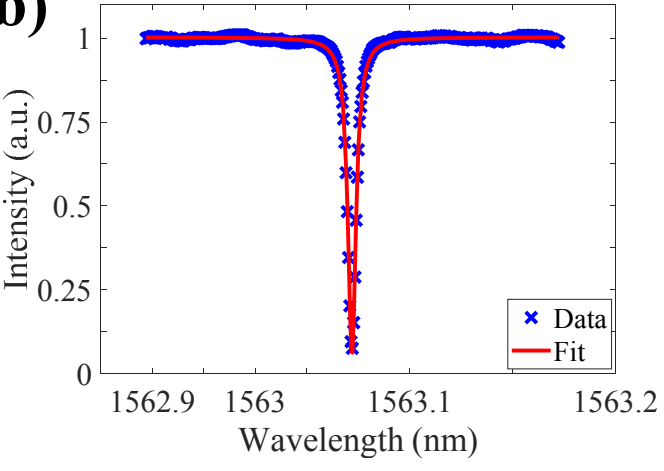

Figure 2. $\mathrm{Al}_{2} \mathrm{O}_{3}$ microring resonator characterization. (a), TE polarized light transmission spectrum of $\mathrm{Al}_{2} \mathrm{O}_{3}$ microring resonator with a cladding of air. (b), Single resonance dip from (a) together with its Lorentzian fit $\left(\mathrm{R}^{2}=0.9973\right)$.

Table 1. Summary of optical characterization of microring resonators at wavelength $\lambda$ of $1565 \mathrm{~nm}$.

\begin{tabular}{|c|c|c|c|}
\hline Type & F (-) & Q (-) & Loss $(\mathrm{dB} / \mathrm{cm})$ \\
\hline TE air & $239 \pm 8$ & $3.2 \pm 0.2 \times 10^{5}$ & $0.60 \pm 0.04$ \\
\hline TM air & $71 \pm 4$ & $9.6 \pm 0.5 \times 10^{4}$ & $2.00 \pm 0.19$ \\
\hline TE water & $44 \pm 6$ & $5.9 \pm 0.8 \times 10^{4}$ & $4.0 \pm 0.6$ \\
\hline TM water & $33 \pm 4$ & $4.5 \pm 0.5 \times 10^{4}$ & $5.7 \pm 0.7$ \\
\hline
\end{tabular}

\section{BULK REFRACTIVE INDEX SENSING}

The $\mathrm{Al}_{2} \mathrm{O}_{3}$ microring resonator can be used as a sensor by varying its local dielectric environment while simultaneously monitoring the induced wavelength shift of the resonances. Such a variation can be achieved by changing the refractive index of the cladding and is called bulk refractive index sensing. Here, deionized (DI) water was used as the top cladding of the sensor and its refractive index was altered by adding and dissolving different concentrations of salt into it. The refractive index of DI water can be increased by $0.0018 \mathrm{RIU}$ per percentage mass fraction of $\mathrm{NaCl}$ in DI water. Solutions were prepared from $0.0 \mathrm{wt} / \%$ up to $0.3 \mathrm{wt} \% \mathrm{NaCl}$ in steps of $0.05 \mathrm{wt} \%$. These were then flown through the microfluidic device over the sensor at a rate of $200 \mu \mathrm{l} / \mathrm{min}$. During the bulk refractive index sensing experiment the flow alternated between the DI water and the solutions with mass fractions of $\mathrm{NaCl}$. At the same time the transmission spectra of the sensor was recorded repeatedly. From these, the resonance dips were extracted, normalized by a background subtraction and fitted with a Lorentzian function to determine the wavelength of the resonances in real time. This was done for both TE and TM polarizations. From this experiment, the $S$ and LOD of the sensor were determined. 

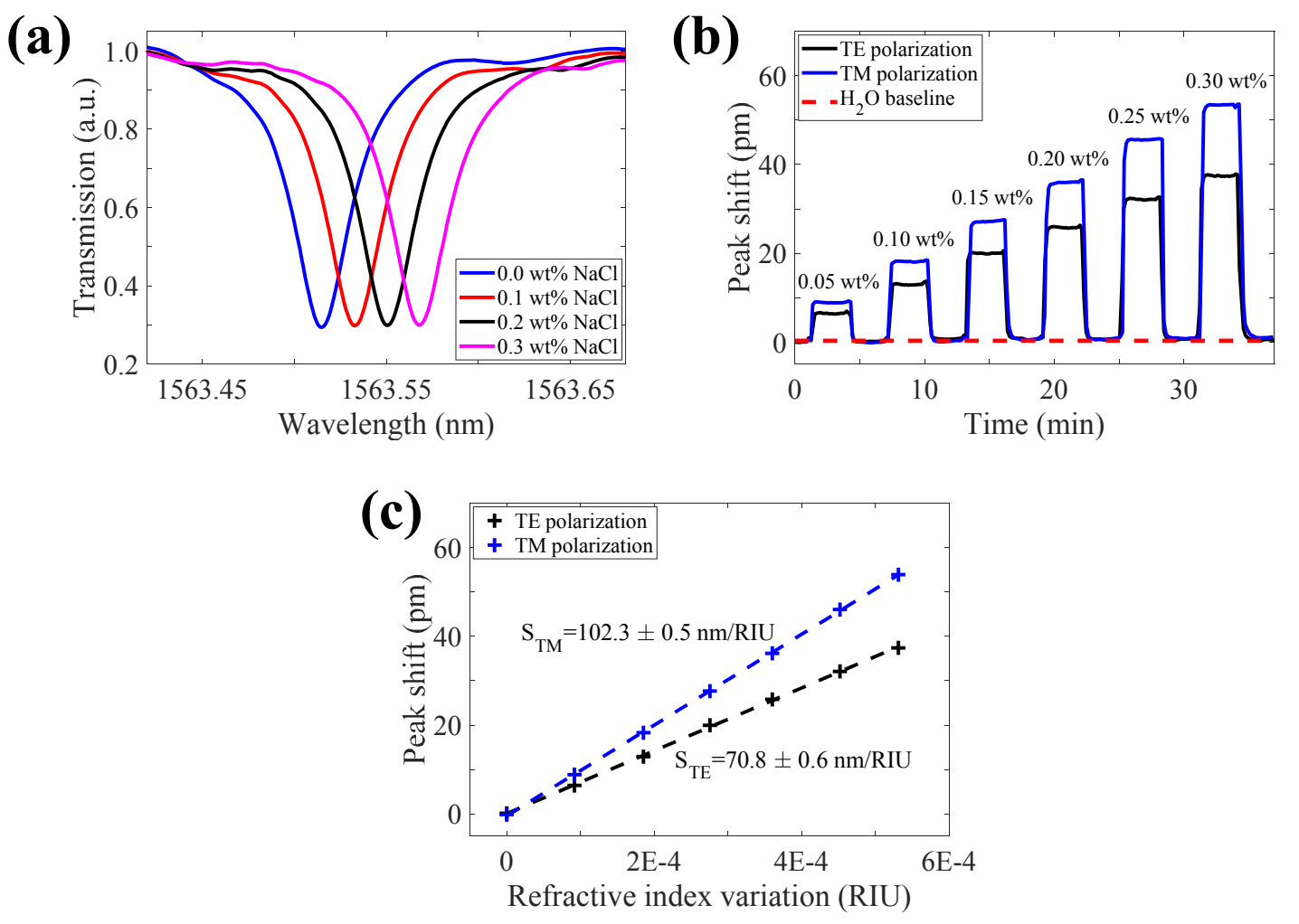

Figure 3. Sensorgram of bulk refractive index sensing with the $\mathrm{Al}_{2} \mathrm{O}_{3}$ microring resonator sensor. (a), Resonance wavelength shift due to varying the concentration of $\mathrm{NaCl}$ in $\mathrm{Di}$ water for $\mathrm{TM}$ polarized light. (b), Real time monitoring of resonance wavelength shift by flowing water with varying concentrations of dissolved $\mathrm{NaCl}$. (c), Bulk refractive index slope sensitivities of the $\mathrm{Al}_{2} \mathrm{O}_{3}$ microring resonator.

The $\mathrm{NaCl}$ solutions induced a shift of the resonance wavelength, as shown in Figure 3a. By monitoring the fitted resonance wavelength over time the sensorgram of the device was constructed and it is displayed in Figure $3 \mathrm{~b}$. By altering the flow between DI water (baseline) and the mass fractions of $\mathrm{NaCl}$ the output of the sensor varies between the baseline and increasing resonance wavelength shifts due to the increased refractive index of the top cladding. The shift is stronger for TM polarized light since the evanescent tail of the modal field extends more into the sensing region. From the sensorgram the slope sensitivity $S$ was determined, as shown in Figure 3c. The resonance wavelength shift was averaged over 10 data points and the measurement uncertainty $\varepsilon$ is defined as the standard deviation thereof. Using Equations (1) and (2) the $S$ and LOD were determined and their values are given in Table 2. The sensor has the best performance for TM polarized light with $S=102.3 \pm 0.5 \mathrm{~nm} / \mathrm{RIU}$ and $\mathrm{LOD}=1.6 \times 10^{-6} \mathrm{RIU}$, making its performance comparable with the silicon-based ring resonator sensing technologies. Due to the better bulk refractive index sensing performance of the sensor at TM polarization, the following biosensing experiments were performed in this polarization.

Table 2. Summary of bulk refractive index sensing performance parameters.

\begin{tabular}{|c|c|c|c|}
\hline Polarization & $\varepsilon(\mathrm{pm})$ & $S(\mathrm{~nm} / \mathrm{RIU})$ & LOD (RIU) \\
\hline $\mathrm{TE}$ & 0.07 & $70.8 \pm 0.6$ & $3.0 \times 10^{-6}$ \\
\hline $\mathrm{TM}$ & 0.05 & $102.3 \pm 0.5$ & $1.6 \times 10^{-6}$ \\
\hline
\end{tabular}




\section{BIOMARKER BIOSENSING}

The $\mathrm{Al}_{2} \mathrm{O}_{3}$ microring resonator can be operated as a biosensor by monitoring the induced resonance wavelength shift upon biomarker binding to its surface. For the biosensing experiment the monoclonal antibody and protein pair S100A4 was chosen because of its involvement in human tumor metastasis ${ }^{12}$. First, the surface of the sensor needs to be prepared with the proper biochemistry. The $\mathrm{Al}_{2} \mathrm{O}_{3}$ surface was functionalized by applying heptane with $500 \mu \mathrm{M} 6-$ phosphonohexanoic acid. The formed carboxylate layer was then activated immersing in $25 \mathrm{mM}$ 1-Ethyl-3-(3dimethylaminopropyl)carbodiimide (EDC) and $50 \mathrm{mM}$ 1-Hydroxy-2,5-pyrrolidinedione (NHS), both in 2-(Nmorpholino)ethanesulfonic acid (MES) buffer. Next, the surface is thoroughly rinsed with $10 \mathrm{mM}$ phosphate buffered saline (PBS) buffer followed by DI water.

After applying the surface biochemistry the sensor was prepared for the biosensing experiment. First, a flow of PBS buffer was applied to establish a stable baseline of the resonance wavelength. Then, PBS buffer containing $50 \mu \mathrm{g} / \mathrm{ml}$ monoclonal antibodies was flown over the sensor at a flow of $80 \mu 1 / \mathrm{min}$, as shown in Figure 4a. By employing the same resonance tracking method described in the previous section, the binding of the antibodies to the surface of the sensor was monitored real-time. The introduction of the antibodies to the sensor was accompanied by a strong shift of the resonance. After 15 minutes the shift saturated, indicating the formation of a monolayer of antibodies on the microring resonator. After switching back to PBS the shift remained stationary, indicating that the formed layer was stable and that the antibodies bound strongly to the sensor. To prevent non-specific protein adsorption to its surface, the sensor was exposed to a $1 \mathrm{wt} \%$ solution of bovine serum albumin (BSA) in PBS, which was flown over the sensor for 10 minutes as shown in Figure 4b. This resulted only in a small shift, since most of the surface of the sensor was already covered with the antibodies. Finally, PBS containing the biomarkers was flown over the device. This was done in 9 steps of increasing concentration, starting from $6 \mathrm{nM}$ up to $1.6 \mu \mathrm{M}$ biomarker content, as shown in Figure 4c. From $12 \mathrm{nM}$ on a clear shift was visible that indicated that the biomarkers bind to the antibodies on the sensor. This shift increased for higher concentrations up to $400 \mathrm{nM}$. At this concentration most of the antibodies have already captured biomarkers and the sensor was almost fully saturated, resulting in small shifts at even higher concentrations.
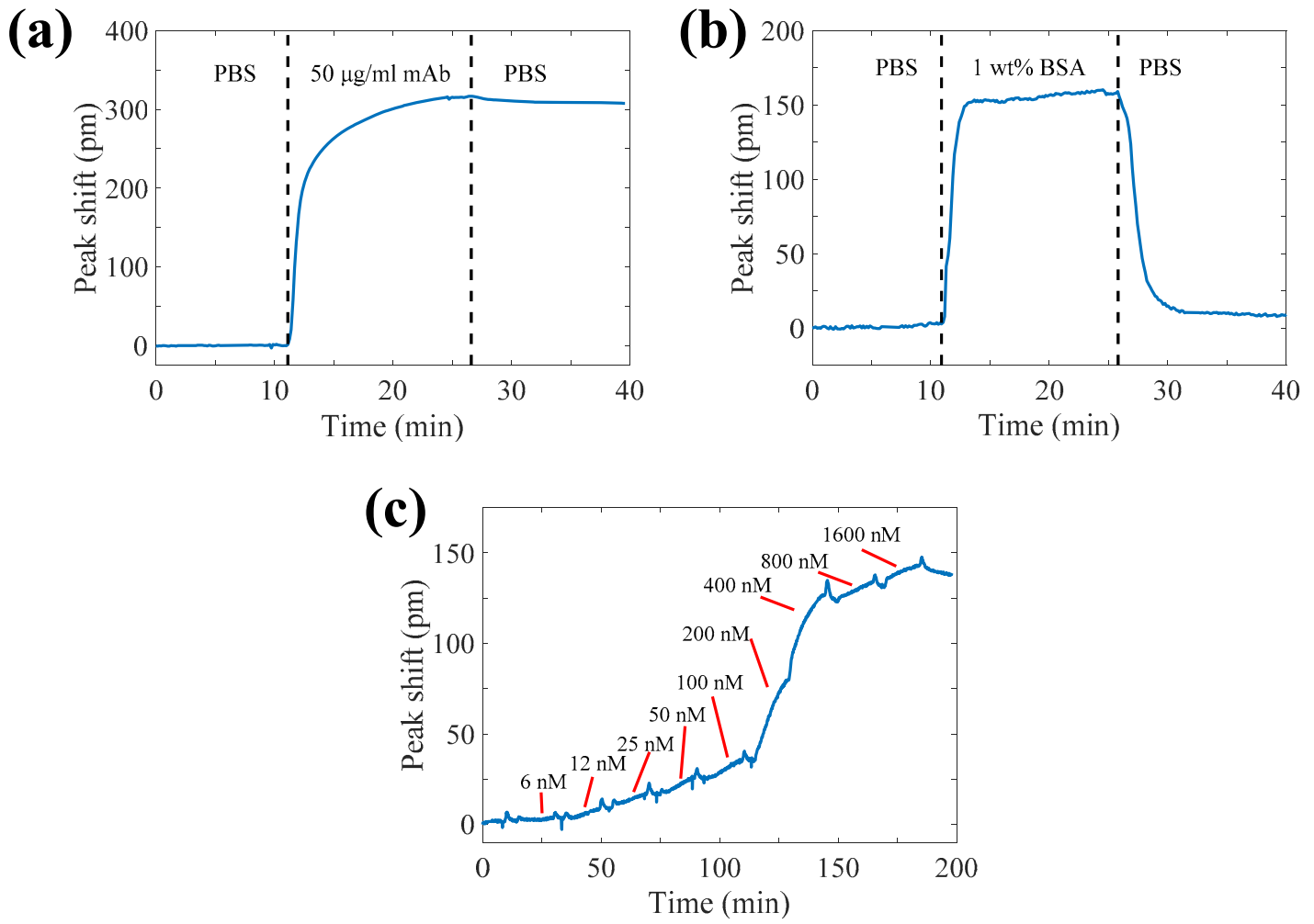

Figure 4. Sensorgram of biomarker detection with the $\mathrm{Al}_{2} \mathrm{O}_{3}$ microring resonator sensor. (a), Immobilization of human monoclonal antibody (mAb) S100A4 onto the surface of the sensor. (b), Surface blocking with BSA. (c), Detection of protein binding to the antibodies. 


\section{CONCLUSION}

$\mathrm{An}_{2} \mathrm{O}_{2} \mathrm{O}_{3}$ microring resonator sensor was realized for bulk refractive index and biosensing applications. The sensor was designed and fabricated for operation in an aqueous environment at a central wavelength of $1550 \mathrm{~nm}$. Optical characterization showed that the devices with a cladding of air can have $Q$ factors up to $3.2 \times 10^{5}$, corresponding with waveguide propagation losses of $0.6 \mathrm{~dB} / \mathrm{cm}$. Upon submerging the devices in water, the $Q$ factors reduced to $59 \times 10^{3}$ and $45 \times 10^{3}$ for TE and TM polarization respectively. The $Q$ factors were reduced by increased waveguide propagation losses due the strong light absorption of water around $1550 \mathrm{~nm}$. Bulk refractive index sensing was performed on this platform and a sensitivity of $102.3 \mathrm{~nm} / \mathrm{RIU}$ and limit of detection of $1.6 \times 10^{-6} \mathrm{RIU}$ was achieved for operation with TM polarized light. Finally, by applying appropriate surface functionalization to the surface of the $\mathrm{Al}_{2} \mathrm{O}_{3}$ microring resonator, human monoclonal antibodies S100A4 were immobilized on the sensor. These were used to capture and bind protein biomarker S100A4 to the sensor resulting in a shift of resonance wavelength. A smallest concentration of $12 \mathrm{nM}$ protein biomarker was detected.

These results demonstrate the viability of using the $\mathrm{Al}_{2} \mathrm{O}_{3}$ waveguide technology for sensitive bulk refractive index sensing and the detection of specific protein biomarkers down to $\mathrm{nM}$ concentrations. Furthermore, this platform offers features that allow extending the sensing technology to more advanced sensors with better performance, such as operation at wavelengths outside the water absorbance window such as at $1 \mu \mathrm{m}$ and the incorporation of rare-earth ions for active materials and lasing based sensors for easier and more sensitive detection principles.

\section{ACKNOWLEDGEMENTS}

This project has received funding from the European Union's Seventh Programme for research, technological development and demonstration under grant agreement No 634928

\section{REFERENCES}

[1] Sun, Y. and Fan,X., "Optical ring resonators for biochemical and chemical sensing," Anal. Bioanal. Chem. 399(1), 205-211 (2011).

[2] Iqbal, M., Gleeson, M. A., Spaugh, B., Tybor, F., Gunn, W. G., Hochberg, M., Baehr-Jones, T., Bailey, R. C. and Gunn, L. C., "Label-free biosensor arrays based on silicon ring resonators and high-speed optical scanning instrumentation," IEEE J. Quantum Electron. 16(3), 654-661(2010).

[3] Heideman, R., Hoekman, M. and Schreuder, E., "Triplex-based integrated optical ring resonators for lab-on-achip and environmental detection," IEEE J. Quantum Electron. 18(5), 1583-1596 (2012).

[4] Samusenko, A., Gandolfi, D., Pucker, G., Chalyan, T., Guider, R., Ghulinyan, M. and Pavesi, L., "A SiON microring resonator-based platform for biosensing at $850 \mathrm{~nm}$," J. Lightwave Technol. 34(3), 969-977 (2016).

[5] Bradley, J. D. B., Ay, F., Wörhoff, K. and Pollnau, M., "Fabrication of low-loss channel waveguides in $\mathrm{Al}_{2} \mathrm{O}_{3}$ and $\mathrm{Y}_{2} \mathrm{O}_{3}$ layers by inductively coupled plasma reactive ion etching", Appl. Phys. B 89(2), 311-318 (2007).

[6] Este, G. and Westwood, W. D., "Reactive deposition of low loss $\mathrm{Al}_{2} \mathrm{O}_{3}$ optical waveguides by modified dc planar magnetron sputtering," J. Vac. Sci. Technol. A 2(3), 1238-1247 (1984).

[7] Bradley, J. D. B., Agazzi, L., Geskus, D., Wörhoff, K. and Pollnau, M., "Gain bandwidth of $80 \mathrm{~nm}$ and $2 \mathrm{~dB} / \mathrm{cm}$ peak gain in $\mathrm{Al}_{2} \mathrm{O}_{3}: \mathrm{Er}^{3+}$ optical amplifiers on silicon," J. Opt. Soc. Am. B 27(2), 187-196 (2007).

[8] Bradley, J. B. D., Stoffer, R., Agazzi, L., Ay, F., Wörhoff, K. and Pollnau, M., "Integrated $\mathrm{Al}_{2} \mathrm{O}_{3}: \mathrm{Er}^{3+}$ ring lasers on silicon with wide wavelength selectivity," Opt. Lett. 35(1), 73-75 (2010).

[9] Bernhardi, E. H., van der Werf, K. O., Hollink, A. J., Wörhoff, K., Ridder, R. M., Subramaniam, V. and Pollnau, M., "Intra-laser-cavity microparticle sensing with a dual-wavelength distributed-feedback laser," Laser Photon. Rev. 7(4), 589-598 (2013).

[10] Bogaerts, W., De Heyn, P.,Van Varenbergh, T., De Vos, K., Selvaraja, S. K., Claes, T., Dumon, P., Van Thourhout, D. and Baets, R., "Silicon microring resonators," Laser Photon. Rev. 27(1), 47-73 (2012).

[11] White, I. M. and Fan, X., "On the performance quantification of resonant refractive index sensors," Opt. Expr. 16(2), 1020-1028 (2008).

[12] Garett, S. C., Varney, K. M., Weber, D. J., Bresnick, A. R., "S100A4, a mediator of metastasis," J. Biol. Chem. 281(2), 677-680 (2006). 